\title{
LA - $M$ BILABIAL EN POSICIÓN FINAL ABSOLUTA EN EL ESPAÑOL HABLADO EN MÉRIDA, YUCATÁN (MÉXICO)
}

El español de la península de Yucatán, México, es de sumo interés lingüístico por su estado de intenso polimorfismo, su gran variedad de peculiaridades fonéticas y su relación de coexistencia con la lengua maya ${ }^{1}$. Un ejemplo de su polimorfismo y sus peculiaridades es la situación de las nasales en posición final absoluta, especialmente ante pausa, donde existen más alófonos posi-

${ }^{1}$ Se han escrito varios estudios que describen las peculiaridades del español de Yucatán. Los de los años anteriores a los sesenta suelen ser los menos sistemáticos: Aloys R. NyKL, "Notes on the Spanish of: Yucatán, Veracruz and Tlaxcala", $M P h, 27$ (1929-1930), 451-460; MANuel G. Revilla, "Provincialismos de fonética en México", en Pedro Henríquez Ureña (ed.), El español en Méjico, los Estados Unidos y la América Central, BDH, t. 4, 1938, pp. 199-206; Antonio Mediz Bolıo, Interinfluencia del maya con el español de Yucatán, Zambá, Mérida, Yucatán, 1951; Alfredo Barrera Vásouez, "Mayismo y voces mayas en el español de Yucatán”, Investigaciones Lingüisticas, 4 (1937), 9-35; y por el mismo autor, "La lengua española de Yucatán", Enciclopedia Yucatanense, 6 (1946), 341-375. El más completo tratado de esa época es de Víctor M. Suárez, El español que se habla en Yucatán, Díaz Massa, Mérida, Yucatán, 1945. Los estudios más recientes tienden a ser más sistemáticos y más completos: Manuel Alvar, "Nuevas notas sobre el español de Yucatán (México)", $I$, 1 (1969), 159-189; Josefina García Fajardo, Fonética del español de Valladolid, Yucatán, UNAM, México, 1984; JUAN M. LOPE BLANCH ha reunido varios trabajos suyos sobre el tema en su libro Estudios sobre el español de Yucatán, UNAM, México, 1987. Entre las peculiaridades que mencionan, las más tratadas son la articulación glotalizada y aspirada de las oclusivas sordas $/ \mathrm{p}, \mathrm{t}, \mathrm{k} /$, y la clausura glotal después de vocal final acentuada o antes de vocal tónica inicial. Otras incluyen la despalatalización de la $/ \tilde{\mathbf{n}} /$ (niña $\rightarrow$ [ninia]) y de la $/ \mathrm{y} /$ intervocálica (silla $\rightarrow$ [siia]), la aspiración y sonorización de la fricativa sorda $\operatorname{velar} / x /($ caja $\rightarrow$ [kaha]), la /s/ sorda en distribución libre con la sonora ante consonante sonora, la articulación oclusiva de $/ b, d, g /$ donde en la lengua estándar aparecen como fricativas, la $/ \mathrm{r} / \mathrm{y} / \mathrm{r} /$ retroflejas $\mathrm{y}$, por supuesto, la pronunciación de $-n$ final como bilabial (pan $\rightarrow$ pam). 
bles que el alveolar [-n] del español estándar. Las posibilidades

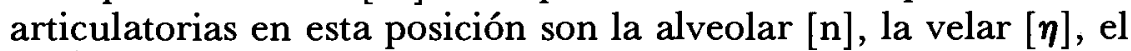
$\emptyset$ fonético acompañado por la nasalización de la vocal anterior ( $(\tilde{v} \emptyset)$ y la labial, que puede ocurrir con varios grados de labialización, desde un ligero acercamiento de los labios en su último momento $\left[\mathrm{n}^{\mathrm{m}}\right]$, a una pronunciación alveolo-bilabial mixta $\left[\begin{array}{c}\mathrm{m} \\ n\end{array}\right]$, hasta una realización plenamente bilabial $[-\mathrm{m}]$.

Las tres primeras soluciones $([n],[\eta], \tilde{v} \emptyset)$ están generalizadas en el mundo hispánico, pero la labializada es bastante limitada fuera de Yucatán, por lo que resulta de particular interés. Fuera de la península yucateca, el fenómeno sólo se da esporádicamente en la República Mexicana². Aunque Luis Flórez y José Joaquín Montes indican que la $-m$ final aparece en la costa colombiana del Pacífico ${ }^{3}$, y también hay noticias del fenómeno en el norte de Argentina, parece que es más intenso en el habla yucateca. Alvar afirma que la - $m$ final "reaparece con una terca constancia en el español yucateco con un carácter bastante aislado dentro del mundo hispánico..." 4 .

García Fajardo, en su estudio del habla de Valladolid, Yucatán, indica que la - $m$ final aparece en el habla de casi todos los informantes, oscilando entre el $5 \%$ y el $40 \%$, aunque en el habla de la mayoría de los informantes aparece con frecuencias menores del $20 \%^{5}$. También indica que la bilabial - $m$ ocurre indistintamente en los diferentes grupos socioculturales, generacionales y de sexo. Los datos descritos por Lope Blanch, recogidos en nueve localidades de la península de Yucatán, también indican que casi todos los informantes yucatecos producen la $-m$ final $^{6}$. En las respuestas de los informantes a los cuestionarios lingüísticos se da una articulación labial en el $30.5 \%$ de las nasales finales, mientras que en las conversaciones espontáneas la realización labial

${ }^{2}$ LOPE BLANCH, en el artículo "La interferencia lingüística: un ejemplo del español yucateco" de su colección Estudios. . ., señala que sólo se han registrado ejemplos de la labialización de $-n$ en $\mathbf{4 6}$ poblaciones mexicanas de las 183 estudiadas que no eran yucatecas. Por lo general sólo se recoge de un solo informante de estos lugares y apenas representa el $5 \%$ de sus articulaciones de nasal final.

${ }^{3}$ LUIS FLóReZ, La pronunciación del español en Bogotá, Instituto Caro y Cuervo, Bogotá, 1951 y José JOAQuín MonTES, "Un rasgo dialectal del occidente de Colombia: -n >-m", en el Homenaje a Fernando Antonio Martinez, Bogotá, 1979, 215-220.

${ }^{4}$ Art. cit., p. 188.

${ }^{5}$ García Fajardo, op. cit.

6 "La interferencia lingüística... ." 
se produce en el $17 \%$ de los casos de nasal final ${ }^{7}$. Esta diferencia del $13.5 \%$ probablemente se deba a que en los cuestionarios las nasales finales de palabra se pronuncian ante pausa total, mientras que en las conversaciones preceden a otras palabras o a pausas muy breves. Los datos de Lope Blanch y García Fajardo indican que la - $m$ final, aun cuando no se encuentra ante pausa total, está bastante difundida en el español yucateco dentro de todos los niveles sociales, edades y sexos, tanto en los bilingües en español y maya como en los monolingües en español.

La coexistencia del español y el maya en la misma región donde aparece con más intensidad la nasal bilabial final ante pausa nos lleva a la posibilidad de que la $-m$ sea resultado de influencia maya. Lope Blanch concluye que la amplia extensión geográfica de - $m$, a pesar de que su aparición fuera de Yucatán es sólo esporádica, desmiente la posibilidad de que dependa sólo del maya; evidentemente el paso $-n>-m$ puede ocurrir a causa de tendencias internas de la lengua. No obstante, sigue siendo posible que el maya haya facilitado tal paso directa o indirectamente ${ }^{8}$. Indirectamente, porque el maya contribuye a la falta de norma lingüística de la región, lo cual propicia el polimorfismo. Dentro de este polimorfismo ha aparecido la $-m$. En cuanto a una influencia más directa, Lope Blanch describe tres condiciones lingüísticas y sociolingüísticas de la península de Yucatán que son las apropiadas para pensar que la lengua maya haya influido en el español en la aparición de - $m$ final: 1) la situación sociolingüística del maya y el español es tal que el maya goza de un alto prestigio y un buen número de hablantes, lo cual permite que hasta las clases dirigentes del área lo acepten; 2) la - $m$ final existe en varias lenguas mayances, y el trato de las nasales finales en el maya yucateco es muy parecido al del español yucateco; 3) la labialización de nasal final ocurre con mayor frecuencia en el territorio yucateco, donde coincidentemente tiene más vigor el maya ${ }^{9}$.

${ }^{7}$ En esta cifra combino la labial y la mixta, $-m$ bilabial, $25 \%+-m / n$ mixta, $5.5 \%=30.5 \%$. La distribución es la siguiente: en los cuestionarios, $-n=20 \%, \eta$ y $\tilde{v} \emptyset=48.6 \%,-m$ y $m / n=30.5 \%$; en las conversaciones, $-n=73 \%, \eta$ y $\tilde{\text { v }}=10 \%,-m$ y $m / n=17 \%$.

8 "La interferencia lingüística...",

${ }^{9}$ En ibid., p. 50, Lope Blanch confirma que "todos los dialectos mayances parecen tener - $m$ final de palabra, y muchos de ellos, también - $n$ en tal posición, aunque en algunos se realiza, entonces, como velar [ $\eta]$; es posible que en lacandón exista confusión o neutralización de $n / m$ finales, si bien en otras hablas mayances se mantiene la distinción fonológica $n / m$ en esa posición 
Lope Blanch ha comentado sobre el gran interés lingüístico de la situación bilingüe en la península yucateca. Dice que "esta peculiar situación es campo abonado para investigaciones lingüísticas -dialectales y sociolingüísticas - de extraordinario interés"10. El fenómeno de la nasal labializada en posición final, y en particular ante pausa, donde hay menos posibilidad de asimilación regresiva, es ciertamente una de las peculiaridades que merecen más atención. Surgen varias preguntas sobre el fenómeno:

1) $\mathrm{Si}$ es de origen maya, ¿qué papel tienen los hablantes bilingües de maya y español en mantener o propiciar el fenómeno en la actualidad?

2) Se ha observado que el habla de las mujeres muchas veces, aunque no siempre, es más conservadora que la de los hombres ${ }^{11}$. $¿$ Se conforma el uso de $-m$ a esta tendencia?

3) ¿Pertenece este fenómeno más a un grupo sociocultural que a otro? Si las clases de más prestigio lo aceptan es más probable que llegue a formar parte de la norma regional, si es que tal norma existe o está en formación. .

4) ¿Está aumentando en frecuencia el fenómeno en las nuevas generaciones o se está perdiendo?

final absoluta". El libro de STePhen Straight sobre el maya yucateco, The acquisition of Maya phonology: variation in Yucatec child language, Garland, New York, 1976, corrobora las indagaciones de Lope Blanch. La explicación de Straight del trato de las nasales en posición implosiva en maya nos permite observar una correspondencia casi completa entre el maya y el español yucateco en este aspecto. Tanto el español como el maya yucateco exhiben la asimilación del punto de articulación de una nasal al final de sílaba hacia el punto articulatorio de una consonante que la sigue. En el maya yucateco, como en el español yucateco, puede aparecer una nasal alveolar [-n], velar [- $\boldsymbol{\eta}]$ o una debilitación o pérdida de la oclusión de la consonante nasal acompañada por una nasalización de la vocal anterior. Existe además la posibilidad de una nasal labial [-m]. Straight indica que cuando se realiza una [-n] alveolar en esta posición siempre representa el fonema alveolar $/ \mathrm{n} /$, mientras que las realizaciones bilabiales $[-m]$, velares $[-\eta]$ o la pérdida de la nasal acompañada por la nasalización de la vocal anterior $/ \tilde{\mathrm{v}} \emptyset /$ son ambiguas y pueden representar cualquiera de los fonemas nasales. Straight menciona también que en el habla de los niños, muchas veces aparecen todas estas realizaciones sin aparente relación con los fonemas subyacentes, ni siquiera cuando se da la [-n] alveolar. En el caso de los adultos hay una neutralización parcial de las nasales mayas en posición final ante pausa, mientras que en muchos niños hay una neutralización total.

10 "Sobre la influencia del maya en el español de Yucatán", en Estudios..., p. 29.

11 Véase Gregorio Salvador, "Fonética masculina y fonética femenina en el habla de Vertientes y Tarifa (Granada)", Orbis, 1 (1952), 19-24. 
El presente estudio pretende acercarse a las respuestas de estas preguntas con un recuento estadístico del fenómeno de la nasal labial final en el habla de 25 personas de Mérida, Yucatán. Se calculará el porcentaje que representa la articulación labial de las nasales en posición final absoluta ante pausa de acuerdo con los parámetros sociolingüísticos de sexo, nivel sociocultural, generación y conocimiento del maya.

\section{La - $M$ final en el habla de MÉrida}

Aunque la - $m$ final se da en toda la península de Yucatán, se escogió la ciudad de Mérida como punto focal de este estudio porque es la capital del estado de Yucatán y el centro cultural y comercial de la península. Por su importancia regional el español que se habla en Mérida puede tener cierto prestigio para el resto de la península y convertirse en norma regional. El español se ha impuesto en Mérida, aunque sin eliminar al maya, por el continuo contacto que existe entre la gente de las zonas rurales, donde el maya se mantiene dominante, y la gente de la capital estatal ${ }^{12}$. También, si se impone algún rasgo diferenciador en el habla de Mérida, ciudad yucateca con mayor comunicación con el resto de la República Mexicana, lo habrá hecho en contra de mucha más presión normalizadora de la que hay en otras ciudades y pueblos de la península. El español de Mérida representa el sendero lógico que tomará el español de la península yucateca. Claro, queda por verse en términos prácticos si el español de Mérida es sustancialmente diferente del que se habla en otras partes de la península.

Originalmente se entrevistó a 34 personas de los dos sexos, de tres divisiones socioculturales basadas en su nivel educativo, ocupación, intereses y conocimiento del mundo, tres generaciones -18 a 29 años, 30 a 49 años y 50 años o más - y con diferentes grados de conocimiento del maya. Todos eran de la ciudad de Mérida o sus alrededores, o habían residido ahí la mayor parte de su vida. Las conversaciones que se grabaron son indicativas de un habla espontánea dirigida. Los temas incluyen intereses y actividades personales y muchas veces algo sobre las leyendas, costumbres y cultura de Yucatán. Se eliminaron del análisis las entrevistas de nueve participantes porque el sonido no permitía una transcripción fonética fiel, o porque hubo pocos ejemplos de /-n/

12 Alvar, art. cit. y SUÁrez, op. cit. 
en posición final absoluta ante pausa (menos de cinco ocurrencias). Los 25 informantes restantes siguen la distribución que se ve en la tabla 1:

\section{TABLA 1}

Distribución de los informantes de acuerdo con sexo, nivel sociocultural, generación y conocimiento del maya

\begin{tabular}{|c|c|c|c|c|c|c|}
\hline \multirow[t]{2}{*}{ Generación } & \multicolumn{3}{|c|}{$\begin{array}{c}\begin{array}{c}\text { Hombres } \\
(n=13)\end{array} \\
\text { Nivel sociocultural }\end{array}$} & \multicolumn{3}{|c|}{$\begin{array}{c}\text { Mujeres } \\
(n=12) \\
\text { Nivel sociocultural } \\
\end{array}$} \\
\hline & $\begin{array}{c}\text { alto } \\
(n=4)\end{array}$ & $\begin{array}{c}\text { med } \\
(n=4)\end{array}$ & $\begin{array}{c}\text { bajo } \\
(n=5)\end{array}$ & $\begin{array}{c}\text { alto } \\
(n=5)\end{array}$ & $\begin{array}{c}\text { med. } \\
(n=5)\end{array}$ & $\begin{array}{c}\text { bajo } \\
(n=2)\end{array}$ \\
\hline $\begin{array}{c}1 \text { (20-29 años) } \\
(\mathrm{n}=6)\end{array}$ & $\#$ & - & + & - & - & \\
\hline $\begin{array}{c}2(30-49 \text { años }) \\
(\mathrm{n}=9)\end{array}$ & $-\quad+$ & \# & $*+$ & $-\quad \#$ & - \# & \\
\hline $\begin{array}{l}3(50 \text { años o } \\
\text { más) }(\mathrm{n}=10)\end{array}$ & \# & - & $+\quad+$ & - & $-\quad *$ & - \# \\
\hline
\end{tabular}

- persona que no sabe maya

\# persona que sabe algunas palabras

* persona que se defiende en maya, pero con algunas dificultades

+ persona que habla $y$ entiende todo en maya

Para cada informante se transcribieron todas las ocurrencias de nasal en posición final absoluta ante pausa. Ya que en este estudio son de interés las realizaciones bilabiales, para simplificar el cálculo se agruparon las posibles realizaciones de la siguiente manera:
$-n$
alveolar
$\eta / \tilde{v} \emptyset$
velar o Ø fonético acompañados por nasalización de la vo- cal anterior
$-\mathbf{m}$
bilabial o mixta con labialización de mayor o menor grado

La nasalización de la vocal anterior a la nasal era general. Para cada informante se calculó la frecuencia relativa de cada una de las posibilidades anotadas arriba, esto es, en el habla de cada persona se dividió el número de ocurrencias de cada tipo de realización entre el número total de nasales en posición final ante pausa. Para cada parámetro sociolingüístico se sacó el promedio de las frecuencias relativas individuales. De esta manera se controlaron los diferentes números de nasales finales en el habla de diferentes individuos y grupos. 
Habiendo visto que la situación lingüística y sociolingüística de Yucatán es propicia para una influencia fonética del maya en las nasales ante pausa, veamos ahora el papel de los bilingües maya/español en este fenómeno. La búsqueda original de informantes se basaba en los criterios de sexo, generación y nivel sociocultural. Después de las entrevistas se preguntó a los informantes si hablaban o entendían maya. Se agruparon las respuestas en cuatro niveles de conocimiento: 0 , no sabe maya; 1 , sabe algunas palabras; 2 , se defiende en maya, pero con algunas dificultades y 3 , habla y entiende todo en maya. Hay que reconocer que este tipo de autoclasificación no siempre es un fiel reflejo de las verdaderas habilidades lingüísticas de los individuos. Además, no se tomó en cuenta la diferencia entre los que lo habían aprendido en casa (la mayoría de los bilingües entrevistados) y los que lo habían aprendido en la escuela (la minoría). Como se ve en la Tabla 1, sólo una de las 12 mujeres informa que se defiende en maya y ninguna dice que habla y entiende todo. Por lo tanto se limita la siguiente exposición sobre la relación actual entre el maya y la $-m$ al habla masculina. A pesar de estas limitaciones, la información recogida puede darnos una idea de la contribución de los bilingües a la frecuencia del fenómeno.

Para determinar si existía una relación entre el conocimiento del maya y la ocurrencia de la nasal bilabial en posición final absoluta ante pausa, se calculó la correlación estadística (coeficiente de Pearson) entre las dos variables. El coeficiente resultante, $.1108, p>.05$, indica la falta de una correlación estadísticamente significativa entre estas dos variables. En otras palabras, un mayor conocimiento del maya no tiende a corresponder ni a más uso de $-m$ ni a menos uso de $-m$ para este grupo de hombres.

Si hoy en día la - $m$ no se oye más en el español de los hablantes bilingües con mayor conocimiento de maya, ies factible que el maya haya tenido alguna influencia directa en la aparición del fenómeno? En esta encuesta todos los informantes son bastante hispanizados, es decir que el español suele ser su lengua dominante, lo cual podría limitar drásticamente la influencia fonética del maya en su español. Esto no quiere decir que no puede haber influencia fonética del maya en el español en alguna época anterior o en un grupo bilingüe en que predomine el maya. En épocas anteriores, y aún actualmente en localidades fuera de Mérida, el maya ha sido la única lengua materna de muchos habitantes, y el aprendizaje del español ha ocurrido en muchos casos en una edad posterior a la pubertad, lo que dificulta la adquisición 
de una pronunciación casi nativa en español. Tales circunstancias podrían ser propicias para la influencia fonética del maya en el español de los bilingües. Si estos hablantes, cuyo maya dominaba fonéticamente su español, tenían el respeto de otros sectores de la población yucateca, ciertos rasgos característicos de su habla, la - $m$ final, por ejemplo, podrían ser adoptados en el habla de los otros sectores. En fin, el que la $-m$ prepáusica no ocurra más frecuentemente en el habla de los bilingües maya/español de Mérida en la actualidad, no quiere decir que en otra época, con otras circunstancias de bilingüismo, el maya no podría haber influido en la aparición de la - $m$ final en el español de los bilingües y después en el de los hablantes dominantes o monolingües en español. Por supuesto, con las limitaciones de la presente encuesta en cuanto al conocimiento del maya, ni siquiera se elimina la posibilidad de que haya tal influencia fonética actualmente en Mérida en hablantes cuyo maya domina su español. De obvia utilidad sería grabar conversaciones en español con hablantes dominantes en maya y averiguar más precisamente su grado de conocimiento del maya y las circunstancias en las que lo aprendieron y lo usan actualmente. Quizás sea difícil encontrar a personas oriundas de la ciudad de Mérida, hablantes maternas de maya, cuyo maya sea más fuerte que su español.

Ya que el fenómeno no parece ser indicador del habla de los bilingües en español y maya, pasaremos a ver si aparece más en algún grupo generacional, sociocultural o de sexo.

La distribución de alófonos para el grupo entero de 25 informantes y para cada sexo se encuentra a continuación en la tabla 2:

TABLA 2

Distribución de nasales en posición final absoluta para el grupo entero, de acuerdo con el sexo

\begin{tabular}{lccc}
\hline & Grupo entero $(n=25)$ & Hombres $(n=13)$ & Mujeres $(n=12)$ \\
\hline$-n$ & .41 & .47 & .34 \\
$-m$ & .41 & .32 & .50 \\
$\tilde{\mathrm{v}} \emptyset /-\eta$ & .18 & .21 & .16 \\
\hline
\end{tabular}

Para el grupo entero la frecuencia de $-n$ y $-m$ es igual: .41. Esto es superior a las frecuencias de - $m$ que encontró García Fajardo en el español de Valladolid, Yucatán ${ }^{13}$. En su estudio el $72 \%$

${ }^{13}$ García Fajardo, $o p$. cit. 
de los entrevistados produjo la bilabial - $m$ con frecuencias menores del 20\%. En las conversaciones espontáneas que describe Lope Blanch el porcentaje es aún menor, el $17 \%$. Se puede atribuir estas diferencias, por lo menos en parte, a que en los datos del presente estudio sólo se tomaron en cuenta las nasales finales ante pausa, mientras que en los datos de García Fajardo y Lope Blanch no se limitan a la posición prepáusica. También puede indicar simplemente que la - $m$ ante pausa es más frecuente en el habla de Mérida que en los otros lugares estudiados.

Volviendo a los datos de la tabla 2 , se ve que la frecuencia $\operatorname{de}[-\eta]$ y $\tilde{v} \emptyset$ es mucho menor que las otras. La frecuencia relativa .18 de estos datos, aunque mayor que el .10 que describe Lope Blanch, no se diferencia demasiado de esta última. Lo interesante son las diferencias entre los sexos. Mientras que el uso de la - $m$ es mucho más común entre las mujeres que entre los hombres (.50 comparado con .32) el habla de los hombres es más estándar en el uso de las nasales finales, ya que recurren a la articulación alveolar estándar más frecuentemente que las mujeres $(.47 \mathrm{com}-$ parado con .34).

El próximo paso es analizar la - $m$ de acuerdo con el parámetro generacional. Como se ve en la tabla 3 , la $-m$ es un rasgo del habla joven. Desde una frecuencia relativa de .34 en la generación de mayor edad, sube a .40 en la generación de en medio hasta aparecer con una frecuencia relativa de .55 en los jóvenes. Estos datos indican una fuerte estratificación de esta articulación en el eje generacional.

TABLA 3

Distribución generacional y sexual de nasales en posición final absoluta ante pausa

\begin{tabular}{cccccccccc}
\hline & \multicolumn{1}{c}{ Grupo entero $(n=25)$} & \multicolumn{3}{c}{ Hombres $(n=13)$} & \multicolumn{3}{c}{ Mujeres $(n=12)$} \\
\hline Generación & $-m$ & $-n$ & $\tilde{\mathrm{v}} \emptyset /-\eta$ & $-m$ & $-n$ & $\tilde{\mathrm{v}} \emptyset-\eta$ & $-m$ & $-n$ & $\tilde{\mathrm{v}} \emptyset /-\eta$ \\
\hline I & .55 & .32 & .13 & .58 & .23 & .19 & .52 & .40 & .08 \\
II & .40 & .39 & .21 & .30 & .41 & .29 & .52 & .37 & .11 \\
III & .34 & .47 & .19 & .20 & .67 & .13 & .48 & .28 & .24 \\
\hline
\end{tabular}

Habiendo visto la diferencia entre el habla masculina y la femenina en cuanto a la $-m$, cabe preguntar si su estratificación generacional se debe más a uno u otro sexo. Con este fin la tabla 3 también contiene la distribución de las nasales en posición final ante pausa para cada sexo dentro del parámetro generacional. Ahí 
se ve que la frecuencia de - $m$ en el habla de las mujeres se ha mantenido relativamente estable en las tres generaciones, aunque la realización alveolar va aumentando y las velares y el $\emptyset$ fonético van disminuyendo en las generaciones más jóvenes. Dentro del eje masculino se ve una fuerte estratificación, progresando en frecuencia la $-m$ desde .20 en la generación de mayor edad, a .30 en la generación de mediana edad hasta .58 en la generación joven. Al mismo tiempo, y al contrario de las mujeres, el alófono alveolar estándar se da cada vez menos, bajando de .67 en la generación mayor a .41 en la de en medio y a .23 en la joven. Estos datos ponen de relieve que son las mujeres las que primero adoptaron la - $m$ en este entorno fonético (o que la han mantenido más del maya), mientras los hombres la han adoptado recientemente, y hasta han pasado a las mujeres en su empleo en la generación joven. Son los hombres los responsables de la estratificación de la articulación bilabial que se ve en el grupo entero. Es interesante notar que el uso de - $n$ y - $m$ en posición final ante pausa es más estándar entre los hombres de la generación mayor, pero es cada vez menos estándar en las generaciones siguientes. Por el contrario, el habla de la generación mayor de las mujeres es menos estándar por su mayor uso de las realizaciones bilabiales, velares y de $\emptyset$ fonético. Aunque se mantiene la frecuencia de $-m$ en las generaciones femeninas más jovenes, su habla es más estándar con el uso creciente de la $-n$ alveolar ${ }^{14}$. Queda por verse si este patrón de $-m$ cada vez más frecuente se mantiene vigente para las futuras generaciones masculinas y si las mujeres acompañan a los hombres en el aumento del empleo de la $-m$.

${ }^{14}$ Aunque en otros ámbitos de habla hispana la - $m$ representaría una característica lingüística innovadora y el uso del alófono alveolar estándar se consideraría rasgo conservador, el uso de los términos "innovadora" y "conservador" en el español yucateco depende de si se acepta que la situación de las nasales finales refleja la fusión de los sistemas fonológicos maya y español. Una mayor frecuencia de - $m$ en las mujeres podría representar -en esta peculiar situación de bilingüismo - el habla más conservadora si se toma en cuenta la fonética de las nasales del maya con su $-m$ final como la pronuncación antigua u original en esta sociedad. Esto se puede hacer si se acepta que la articulación de las nasales finales del español yucateco hoy es realmente una extensión de los hábitos mayas. En este sentido el habla femenina ha sido más conservadora mientras el habla masculina ha sido más innovadora en su aceptación del estándar alveolar. Un nuevo orgullo regional podría otra vez dar ímpetu a la - $m$, que se ve actualmente en su mayor frecuencia en el habla masculina de la generación más joven. Por supuesto que me es imposible apoyar tal hipótesis con los datos que tengo. 
Los resultados del análisis por clases socioculturales se representan en la tabla 4:

TABLA 4

Distribución sociocultural y sexual de nasales en posición final absoluta ante pausa

\begin{tabular}{lccccccccc}
\hline & \multicolumn{1}{c}{ Grupo entero $(n=25)$} & \multicolumn{3}{c}{ Hombres $(n=13)$} & \multicolumn{3}{c}{ Mujeres $(n=12)$} \\
\hline Nivel sociocultural & $-m$ & $-n$ & $\tilde{\mathrm{v}} \emptyset /-\eta$ & $-m$ & $-n$ & $\tilde{\mathrm{v}} \emptyset-\eta$ & $m$ & $-n$ & $\tilde{\mathrm{v}} \emptyset /-\eta$ \\
\hline I (alto) & .35 & .43 & .21 & .27 & .44 & .29 & .42 & .43 & .15 \\
II (med.) & .47 & .37 & .16 & .28 & .57 & .15 & .63 & .21 & .16 \\
III (bajo) & .41 & .42 & .17 & .41 & .41 & .18 & .40 & .44 & .15 \\
\hline
\end{tabular}

Para el grupo entero de entrevistados se nota que es en el habla de la clase media donde aparece $-m$ bilabial más frecuentemente, con un porcentaje de 47 , mientras en la clase alta es donde menos se oye (35\%). El porcentaje en la clase baja está entre los de las otras dos: $41 \%$ de las nasales ante pausa. Atendiendo a las diferencias sexuales entre las tres generaciones, es obvio que son las mujeres de clase media las que más articulan la nasal prepáusica bilabialmente (el $63 \%)$. En la clase media masculina prácticamente no es más frecuente que en la clase alta (el $28 \%$ y el $27 \%$, respectivamente). Entre el sexo masculino, a diferencia del femenino, es en la clase baja donde más se oye la $-m$ (el $41 \%$ ) y no en la clase media. Es notorio que el habla masculina de clase media es la única donde la realización alveolar estándar es la mayoritaria.

Regresando a nuestras preguntas originales, a pesar de que las circunstancias lingüísticas y sociolingüísticas admiten la posibilidad de que la - $m$ final ante pausa en el español de Yucatán sea de origen maya, o que por lo menos haya sido propiciada por el maya, en este estudio del español hablado en Mérida, Yucatán no se relaciona la - $m$ final con los hombres que informan que saben más maya, lo cual no necesariamente indica que no existe - o ha existido en otras épocas - tal relación en hablantes cuya lengua materna es el maya y que aprendieron el español después de la pubertad. Viendo la situación de la - $m$ globalmente, esto es, tomando en cuenta los tres parámetros sociolingüísticos, el fenómeno está más establecido históricamente en el habla femenina, oscilando alrededor del $50 \%$ en las tres generaciones. Sin embargo, es en la clase media femenina donde más se oye. Entre los hombres son los jóvenes y la clase baja quienes más la realizan. 
A pesar de que se ha caracterizado el habla femenina como más conservadora que la masculina, parece que en el habla de Mérida esta peculiar pronunciación se ha establecido primero en las mujeres. Esto bien podría representar la conservación de un rasgo maya y no una innovación regional. No obstante, se ha visto mucho progreso del fenómeno en el habla masculina, ya que en la generación joven prevalece más la articulación bilabial de nasal ante pausa que las otras posibilidades combinadas. Parece que ahora las mujeres han pasado el ímpetu de esta característica a los hombres. Como frecuentemente ocurre, es en la clase baja donde se da primero una innovación lingüística, y la - $m$ aparece más en el habla de la clase baja masculina. Sin embargo esta articulación es aceptada por la clase alta, lo cual puede facilitar que siga aumentando la frecuencia de la $-m$ final. Todo esto indica que el fenómeno está adquiriendo más popularidad en el español hablado en Mérida. 